\title{
Effects of Lusutrombopag on Post-invasive Procedural Bleeding in Thrombocytopenic Patients with Chronic Liver Disease
}

\author{
Manami Yoshida Ryosuke Tateishi - Shinzo Hiroi - Yoshie Hongo · \\ Masakazu Fujiwara · Yoshitake Kitanishi · Kosuke Iwasaki • \\ Tomomi Takeshima $\cdot$ Ataru Igarashi
}

Received: September 6, 2021 / Accepted: October 15, 2021 / Published online: November 8, 2021

(C) The Author(s) 2021

\begin{abstract}
Introduction: Thrombocytopenia can increase the bleeding risk in patients with chronic liver disease (CLD) undergoing invasive procedures. Prophylactic platelet transfusion (PT) is often performed to increase platelet counts in patients with CLD undergoing invasive procedures to prevent bleeding. Lusutrombopag, a small-molecule thrombopoietin receptor agonist, is expected to be an alternative therapy to
\end{abstract}

Supplementary Information The online version contains supplementary material available at https:// doi.org/10.1007/s12325-021-01965-7.

M. Yoshida $(\bowtie) \cdot$ S. Hiroi · Y. Hongo

Medical Affairs, Shionogi and Co., Ltd., 7F, Tekko Building, 1-8-2 Marunouchi, Chiyoda-ku, Tokyo 100-0005, Japan

e-mail:manami.yoshida@shionogi.co.jp

M. Yoshida · A. Igarashi

Graduate School of Pharmaceutical Sciences, The

University of Tokyo, 7-3-1 Hongo, Bunkyo-ku,

Tokyo 113-0033, Japan

\section{R. Tateishi}

Department of Gastroenterology, The University of Tokyo Hospital, 7-3-1 Hongo, Bunkyo-ku, Tokyo

113-8655, Japan

M. Fujiwara · Y. Kitanishi

Data Science Department, Shionogi and Co., Ltd., 1-8, Doshomachi 3-chome, Chuo-ku,

Osaka 541-0045, Japan prophylactic PT. This study aimed to compare the effects between lusutrombopag and PT.

Methods: Data were obtained from a Japanese administrative database (April 2008-May 2019). Patients aged $\geq 18$ years who underwent planned invasive procedures after the first CLD diagnosis and were observed for $\geq 30$ days prior to invasive procedures were considered eligible. Patients who underwent planned invasive procedures with lusutrombopag prescription at 5-30 days before the procedure were categorized as the lusutrombopag group, whereas those who received PT at 1 day before and/or on the same day as the procedure, without lusutrombopag prescription, were classified as the PT group. Outcomes, including bleeding frequency during

K. Iwasaki · T. Takeshima

Milliman, Inc., 8F, Urbannet Kojimachi Building, 1-6-2 Kojimachi, Chiyoda-ku, Tokyo 102-0083, Japan

\section{A. Igarashi}

Unit of Public Health and Preventive Medicine,

Yokohama City University School of Medicine, 3-9

Fukuura, Kanazawa-ku, Yokohama 236-0004, Japan 
hospitalization and average medical costs (costs for prophylactic treatment and total costs between the day of the invasive procedure and 30 days after the invasive procedure), were compared between the groups after matching. Results: Among 738,878 patients with CLD, 379 cases for each group were identified after matching. The incidence of bleeding events was lower in the lusutrombopag group than in the PT group $(3.7 \%$ vs. $8.2 \%, p<0.001)$. Average medical costs were lower in the lusutrombopag group than in the PT group (\$6667 as of August 2021 vs. $\$ 7170, p=0.011$ ).

Conclusion: Lusutrombopag is suggested to be effective as a prophylactic treatment for bleeding prevention in patients with CLD undergoing planned invasive procedures.

Keywords: Thrombopoietin receptor agonist; Platelet transfusion; Thrombocytopenia; Liver cirrhosis; Radiofrequency ablation; Hemorrhage; Rescue for bleeding; Medical costs; Length of stay; Real world data

\section{Key Summary Points}

Why carry out this study?

Patients with chronic liver disease (CLD) often require medical and/or surgical diagnostic and therapeutic procedures. Thrombocytopenia is a major complication in these patients, as it increases the bleeding risk during invasive procedures

Prophylactic platelet transfusion (PT) is often performed to increase platelet counts for bleeding prevention. However, PT has several potential risks of side effects and entails a high burden on patients, including hospitalization and intensive care. Lusutrombopag, a small-molecule thrombopoietin receptor agonist, is expected to be an alternative therapy to prophylactic PT

This study compared the effects between lusutrombopag and PT in preventing bleeding and reducing patient burden

\section{What was learned from the study?}

The incidence of bleeding events was lower in the lusutrombopag group than in the PT group (3.7\% vs. $8.2 \%, p<0.001)$. Average medical costs were lower in the lusutrombopag group than in the PT group ( $\$ 6667$ as of August 2021 vs. $\$ 7170$, $p=0.011)$

Lusutrombopag is suggested to be effective as a prophylactic treatment to prevent bleeding in patients with CLD undergoing planned invasive procedures

\section{INTRODUCTION}

Patients with chronic liver disease (CLD) often require invasive procedures, including biopsy and therapeutic procedures for complications and for treatment of hepatocellular carcinoma. Thrombocytopenia in peripheral blood is a major complication in patients with CLD $[1,2]$, which can increase the bleeding risk in patients undergoing invasive procedures. For patients at high bleeding risk due to low platelet counts $(<50,000 / \mu \mathrm{l})$, treatment to increase platelet counts is recommended prior to invasive procedures [2, 3]. Platelet transfusion (PT) is a standard treatment to increase platelet counts prior to procedures for patients at risk for bleeding. However, PT has several potential risks of side effects and entails a high burden on patients, including hospitalization and intensive care [3]. In addition, the safety measure and proper use of the platelet preparations are complicated owing to their short lifetime and the difficulty in storage management in medical facilities [4]. Furthermore, the effects of PT on increasing platelet counts [5] and reducing bleeding events [6] remain uncertain.

Lusutrombopag was first approved for the treatment of thrombocytopenia in patients with CLD scheduled to undergo an invasive procedure in Japan in 2015 and then in the US in 2018. Later, it was also approved for the treatment of severe thrombocytopenia in these 
patients in Europe in 2019. It is an orally administered, small-molecule thrombopoietin receptor agonist that induces proliferation and differentiation of human bone marrow hematopoietic progenitor cells to megakaryocyte cellular lineage, leading to an increased platelet count $[5,7]$. According to the Japanese package insert, the administration of $3 \mathrm{mg}$ lusutrombopag once daily for 7 days should begin approximately 8-13 days before the scheduled date of the invasive procedure [8]. Recently, the 2020 Japanese guidelines recommended the administration of a thrombopoietin receptor agonist prior to planned invasive procedures for thrombocytopenic patients with hepatic cirrhosis [9]. Considering its fewer side effects and ease of use, lusutrombopag is expected to be an alternative therapy to PT. In placebo-controlled, randomized, double-blind trials, lusutrombopag showed a significantly higher efficacy than placebo in terms of preventing the need for PT $[5,7,10]$. Superiority in maintaining platelet counts was also shown among patients who received lusutrombopag without PT compared with those who received placebo and PT $[5,7,10]$. A study using Japanese real world data reported that the effects of lusutrombopag in terms of increasing and maintaining platelet counts were superior to those of PT [11]. In that study, 90 patients who received lusutrombopag and 110 patients who received PT were initially included, and 26 patients in each group were selected by propensity score matching for analysis.

When considering lusutrombopag as an alternative therapy to the prophylactic administration of platelet preparations in clinical practice, the effects with respect to the prevention of bleeding and reduction of patient burden including hospitalization, which are considerable issues when treating with PT, are crucial. The efficacy of lusutrombopag in preventing bleeding events has not been evaluated in placebo-controlled clinical trials. This is because the lower frequency of events associated with invasive procedures for which lusutrombopag administration is approved necessitates a large sample size, which makes it impossible to perform clinical trials with bleeding events as an endpoint. Although a decrease in bleeding events as adverse events has been reported $[5,10]$ and there is an observed trend toward a reduction in bleeding events with lusutrombopag than with placebo/ PT, the evidence is insufficient to conclude that the preventive effect of lusutrombopag against bleeding is superior to that of PT. Additionally, an improvement in patient burden has not yet been evaluated.

In this study, to overcome the difficulty in evaluating the effects in clinical trials, a Japanese nationwide hospital-based administrative database was used to investigate and compare the effects of lusutrombopag and PT as prophylactic treatments to increase platelet counts in patients with CLD undergoing planned invasive procedures. The incidences of bleeding events and rescue therapy for bleeding, as well as the medical costs and length of hospital stay, were compared between thrombocytopenic patients receiving lusutrombopag and those receiving PT at a certain time associated with planned invasive procedures.

\section{METHODS}

\section{Study Design and Setting}

This retrospective cohort study was based on administrative data from hospitals that adopted the Japanese Diagnosis and Procedure Combination (DPC) fixed-payment reimbursement system, also known as DPC hospitals. The database was provided by Medical Data Vision Co., Ltd. (Tokyo, Japan). The database contains claims data of 400 hospitals, which account for $23 \%$ of acute care hospitals, and includes data from 30 million patients [12].

The study period was defined as the entire data collection period, which was from April 2008 to May 2019. The observation period for each patient was defined as the period between the first record and last record of any medical practice in the database.

This study was approved by the Ethics Committee of the University of Tokyo. All procedures followed were in accordance with the ethical standards of Ethical Guidelines for Medical and Health Research Involving Human 
Subjects by the Ministry of Education, Culture, Sports, Science and Technology and the Ministry of Health, Labour and Welfare and with the 1975 Declaration of Helsinki, as revised in 2008. As the database consisted of anonymized data collected for secondary use, informed consent was not required.

\section{Patients}

Patients with CLD who received at least one planned invasive procedure with administration of either lusutrombopag or PT after the first CLD diagnosis were included. Detailed criteria for patient identification for the analyses are shown in Fig. 1. Patients with CLD were defined as those having at least one definitive diagnosis of any type of CLD, including viral hepatitis, autoimmune hepatitis, toxic/drug-induced hepatitis, alcoholic hepatitis, and non-alcoholic steatohepatitis based on the disease name (Supplementary Material Table S1). The planned invasive procedures were defined by the procedure name, which were categorized into 18 types (Supplementary Material Table S2). The procedures were defined as those without record of an add-on fee for emergency (Supplementary Material Table S3). The day of the first CLD diagnosis was defined as the earliest date between the start date of treatment as an outpatient and that of hospitalization with a definitive CLD diagnosis.

The patients identified above were divided into the lusutrombopag group and the PT group according to planned invasive procedures. The lusutrombopag group included cases in which patients initiated a prescription of a course of treatment with lusutrombopag, which was defined by the generic name, lusutrombopag, between 5 and 30 days prior to the invasive procedure. The PT group included cases in which patients received treatment with PT, which was defined as B02D8 by the Anatomical Therapeutic Chemical (ATC) code by the European Pharmaceutical Market Research Association [13], 1 day before and/or on the same day as the invasive procedure, with no prescription of lusutrombopag within 30 days before the procedure. The treatment with lusutrombopag and that with PT provided during the period described above were considered prophylactic treatments. Platelet counts were only available in a limited number of patients in the database, and we were not able to determine patients with thrombocytopenia among all patients. Therefore, we assumed that patients who received treatment with lusutrombopag or PT for planned invasive procedures were thrombocytopenic.

Outcomes were compared between the lusutrombopag and PT groups after matching the groups based on the characteristics of cases. Groups were matched by invasive procedure cases using the following process. First, the cases in the lusutrombopag group were stratified into cells by the following variables: age at the time of the invasive procedure, type of invasive procedure, number of days from hospital admission to the invasive procedure, presence of severe liver disease (SLD), and medical costs at 1 month prior to the invasive procedure (excluding the costs for lusutrombopag or PT). SLD was defined as a definitive diagnosis of liver cancer, defined by the International Statistical Classification of Diseases and Related Health Problems 10th Revision (ICD-10) [14] as C22.0 or decompensated cirrhosis defined by the disease name (Supplementary Material Table S4) [15]. Second, the cases in the cells having those from both the lusutrombopag group and the PT group were identified as the target cases. Third, the identified cases were weighted by each cell as follows: the weighted value of each case in the lusutrombopag group was 1 , and the weighted value of the case in the PT group was calculated as the number of cases in the lusutrombopag group divided by the number of cases in the PT group in the corresponding cell. For example, if there were two cases from the lusutrombopag group and three cases from the PT group, the value of one case in the PT group was calculated to be $2 / 3$.

\section{Outcomes}

The primary outcome was the incidence of bleeding events, which was the percentage of cases in which patients had diagnoses related to 


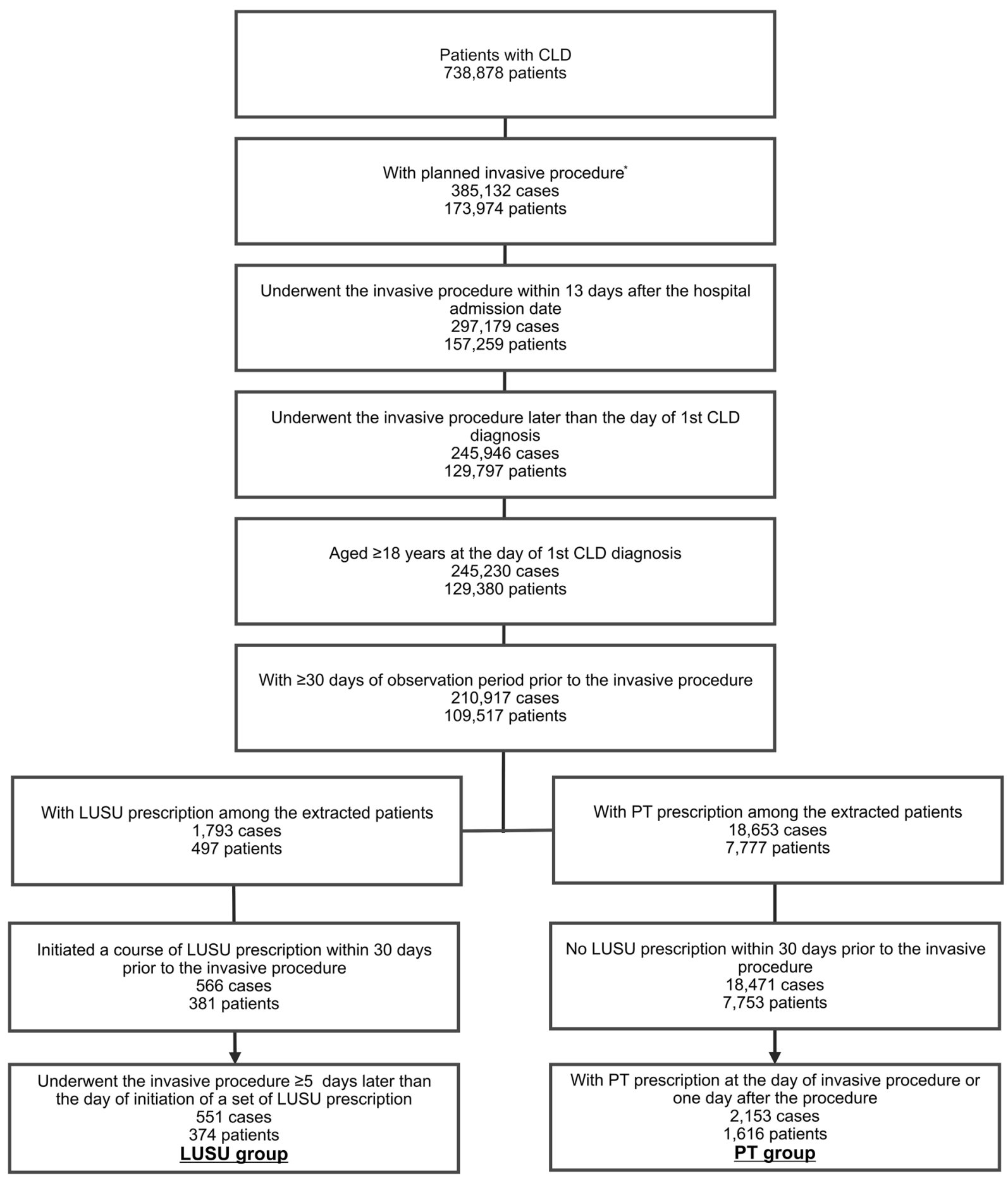

Fig. 1 Flow chart showing patient identification for the analyses. The numbers of patients, invasive procedure cases, and prescriptions identified in each step are described. *Planned invasive procedure, invasive procedure without an add-on fee for emergency. CLD chronic liver disease, LUSU lusutrombopag, PT platelet transfusion 
bleeding (defined by ICD-10 in Supplementary Material Table S5) during hospitalization for invasive procedures.

The secondary outcomes were the incidence of rescue therapy for bleeding, medical costs, and length of hospital stay. Regarding rescue therapy, the percentages of cases with a prescription of PT, red blood cell transfusion (RBCT), or plasma transfusion (PLASMAT) during the period from 1 day after the invasive procedure until the day of hospital discharge was calculated. RBCT was defined by the generic name, concentrated human red blood cells or washed human red blood cells, and PLASMAT was defined by the ATC code B02D6 (fresh-frozen human plasma) or K03B1 (human plasma protein fraction).

As for medical costs, the average costs per patient were calculated for the following: (1) prophylactic treatments to increase platelet counts prior to planned procedures, which comprised costs for lusutrombopag prescribed within 30 days prior to invasive procedures and those for PT prescribed 1 day before and on the day of planned invasive procedures (hereinafter, prophylactic treatment costs), and (2) total medical costs between the day of the invasive procedure and 30 days after the procedure, from which the costs for PT on the day of the procedure were subtracted (hereinafter, post-invasive procedure costs).

Regarding the length of hospital stay, the average number of total hospitalization days was calculated for planned invasive procedures, followed by the days from the day when invasive procedures were performed until the day of discharge.

\section{Statistical Analyses}

Values for the identified cases before and after matching were calculated for each outcome and were compared between the treatment groups after matching. We considered the sample size to be sufficiently large to assume a normal distribution; thus, we used the two-tailed $z$ test or the test for the difference in proportions to assess between-group differences in the values of characteristics before and after matching as well as the outcomes after matching. A difference with $p<0.05$ was considered to be statistically significant.

A subgroup analysis was conducted, wherein cases in the PT group were limited to those who received treatment with PT at 1 day before the invasive procedure, but not on the same day. Groups were matched by applying the same methodology as in the main analysis and were analyzed in a manner similar to that in the main analysis.

Analyses were performed using SAS version 9.4 (SAS Institute, Cary, NC) and Microsoft Excel 2016 (Microsoft, Redmond, WA).

\section{RESULTS}

A total of 738,878 patients with CLD were identified in the database. Of these, 551 cases (374 patients) were included in the lusutrombopag group, whereas 2153 cases (1616 patients) were included in the PT group (Fig. 1). Patients' characteristics are summarized in Table 1. In the PT group, 361 out of 2153 cases had PT prescriptions at 1 day before the invasive procedure but not on the same day of the procedure.

For matching, 551 cases in the lusutrombopag group were stratified into 348 cells. Among these cells, 192 cells had at least one case of the corresponding PT group. A total of 379 cases in the lusutrombopag group and 736 cases in the PT group were finally identified, which were subsequently weighted as 379 cases for each group. The number of cases according to the type of invasive procedures as basic characteristics is presented in Supplementary Table S6. Differences in variables between the groups disappeared after matching (Supplementary Material Table S7). For the subgroup analysis in which cases in the PT group were limited to those who received treatment with PT at 1 day prior to invasive procedures, there were 156 cases in each group after matching.

The incidence of bleeding events was significantly lower in the lusutrombopag group than in the PT group $(3.7 \%$ vs. $8.2 \%, p<0.001)$ (Fig. 2a). The percentage of all three types of rescue therapy for bleeding was lower in the 
Table 1 Characteristics of cases in each group

\begin{tabular}{llll}
\hline & LUSU group & PT group & $\boldsymbol{p}$ value \\
\hline Number of cases & 551 & 2153 & \\
Age at invasive procedure (years) & & & \\
Average (SD) & $68.7(9.4)$ & $66.9(12.0)$ & \\
Number of days to invasive procedure after hospital admission & $3.6(3.6)$ & \\
Average (SD) & $3.2(3.5)$ & $459,478(630,930)$ & $<001$ \\
Medical costs at 1 month before invasive procedure (Japanese yen) & $43 \%$ & 0.001 \\
Average (SD) & $268,897(230,794)$ & \\
Severe liver disease (\%) & $59 \%$ & & \\
\hline
\end{tabular}

LUSU lusutrombopag, $P T$ platelet transfusion, $S D$ standard deviation

lusutrombopag group than in the PT group (Fig. 2b-d). The difference in PT and RBCT therapy between the groups was statistically significant $(p<0.001$ for both); in contrast, the difference in PLASMAT therapy was not $(p=0.107)$. Furthermore, the subgroup analysis showed that the incidence of bleeding events was lower in the lusutrombopag group than in the PT group $(3.2 \%$ vs. $5.1 \%)$; however, the difference was not statistically significant $(p=0.242)$.

Table 2 presents the average medical costs per patient. The total prophylactic treatment cost was higher in the lusutrombopag group than in the PT group. The average total postinvasive procedure cost was lower in the lusutrombopag group than in the PT group. After including the total prophylactic treatment costs and post-invasive procedure costs, the total cost remained lower for the lusutrombopag group ( $¥ 731,220$ [\$6667], with \$1.00 being equivalent to approximately $¥ 109.67$ as of August 2021) than for the PT group (¥786,357 [\$7170]). The difference in overall costs was statistically significant ( $p=0.011)$. In all cases, the difference in overall costs between the groups was much larger than that after matching ( $¥ 729,046$ [\$6648] in the lusutrombopag group and $¥ 1,375,748[\$ 12,544]$ in the PT group; Supplementary Material Table S8).

With respect to the length of hospital stay, both the average number of total hospitalization days for the invasive procedure and the number of days from the day of the invasive procedure until the day of discharge were significantly smaller in the lusutrombopag group (11.8 days and 9.2 days, respectively) than in the PT group (13.9 days and 11.2 days, respectively) $(p<0.001$ for both; Fig. 3a, b).

\section{DISCUSSION}

To our knowledge, this study is the first to evaluate the preventive effect of lusutrombopag against bleeding events. Previous clinical trials reported that the rates of bleeding-related adverse events after lusutrombopag administration were $14.6 \%$ in Japan [5] and $2.8 \%$ in other countries [10]. In the current study, the rate of bleeding events in the lusutrombopag group was $3.7 \%$, which is within the range of previously reported rates. The gap in the findings between these studies may be attributable to the difference in data sources and small sample size in the Japanese trial. The incidence of bleeding events was lower in the lusutrombopag group than in the PT group. However, although the treatment with PT prescribed 1 day before the invasive procedure and on the day of the invasive procedure was defined as prophylactic treatment, whether the PT provided on the day of the invasive procedure was administered before or after the procedure could not be clarified. Thus, it remains possible that some of the PTs prescribed on the 


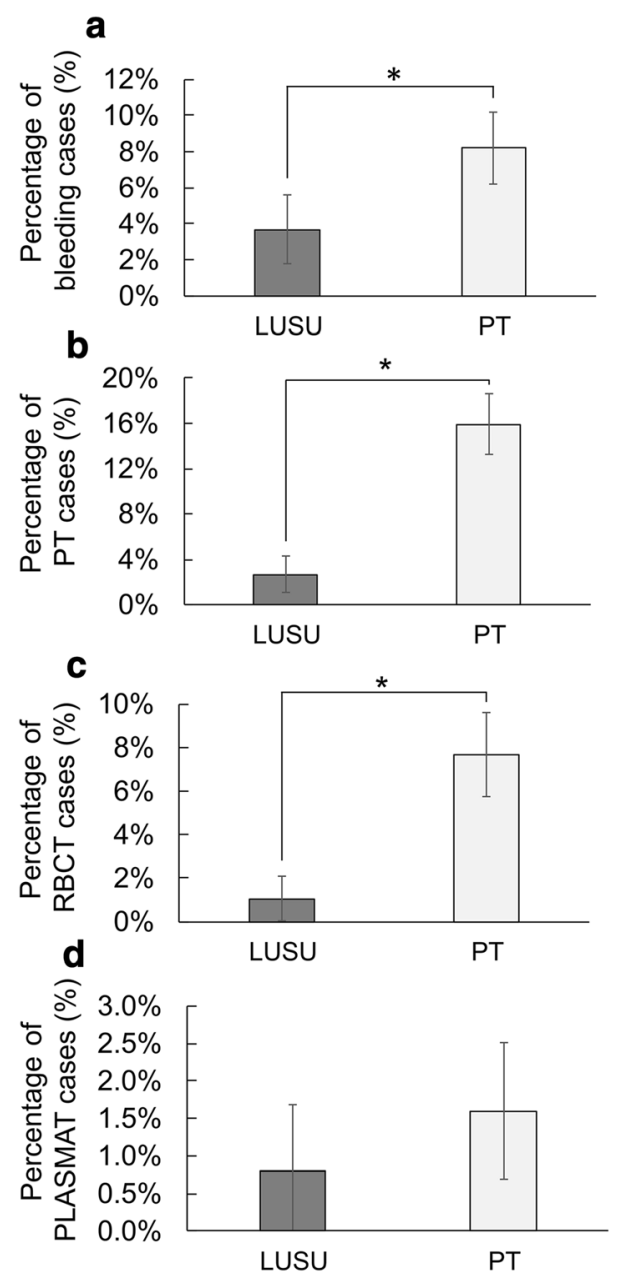

Fig. 2 Percentages of cases with bleeding events (a) and rescue therapy for bleeding, including platelet transfusion (b), red blood cell transfusion (c), and plasma transfusion (d). Error bars represent $95 \%$ confidence intervals. LUSU lusutrombopag, $P T$ platelet transfusion, $R B C T$ red blood cell transfusion, PLASMAT plasma transfusion; ${ }^{*} p<0.05$

day of the invasive procedure were prescribed as rescue therapies for bleeding after the procedure. If the PTs were restricted to only prophylactic PTs, the incidence of bleeding events might have been reduced to some extent in the PT group. We conducted a subgroup analysis in which only the PTs prescribed 1 day before the invasive procedure were included as prophylactic treatments. In the analysis, the rates of bleeding events were $3.2 \%$ and $5.1 \%$ in the lusutrombopag and PT groups, respectively, both of which were lower than those in the main analysis. Considering that prophylactic treatments with PTs are provided on the day of invasive procedure as well, the incidence of bleeding in the subgroup analysis seems to be underestimated, probably leading to an underestimate of the effect of lusutrombopag on the bleeding risk. Although the incidence of rescue therapy for bleeding could also be underestimated, because those administered on the day of the invasive procedure were not included, all three types of rescue therapy showed lower percentages in the lusutrombopag group than in the PT group, and the difference was significant for PT and RBCT compared with those in the PT group. Taken together, the bleeding risk is most likely to be lower with lusutrombopag treatment than with PT.

In all cases and after matching, even though prophylactic treatments to increase platelet counts prior to planned invasive procedures cost more in the lusutrombopag group because of the price of lusutrombopag itself, the overall costs including post-invasive procedure costs were less in the lusutrombopag group than in the PT group. In all cases, post-invasive procedure costs were much higher, approximately $¥ 580,000 \quad$ (\$5289) higher than those after matching in the PT group. This was because a substantial proportion of patients with high medical costs received PT as a prophylactic treatment.

In addition, the length of hospital stay (both the number of total hospitalization days and the number of days from the day of the invasive procedure until the day of discharge) was significantly shorter in the lusutrombopag group than in the PT group. This result could contribute to a reduction in patient burden and medical costs.

In this study, higher effects of lusutrombopag than those of PT in reducing the bleeding risk, medical costs, and length of hospital stay were indicated in patients matched to those who were prescribed lusutrombopag. This does not necessarily mean that lusutrombopag could have been effective for all patients who had been prescribed PT. It shows that patients who did not have similar characteristics in another treatment group were excluded through the process of matching. Thus, the effects of treatments on the excluded patients could not be 
Table 2 Medical costs after matching in each group

\begin{tabular}{lll}
\hline & LUSU group & PT group \\
\hline Number of cases & 379 & 379 \\
(1) Prophylactic treatment costs & \\
LUSU prescribed within 30 days before the invasive procedure $^{\mathrm{a}}$ & & \\
PT at 1 day before the invasive procedure & 109,020 & 0 \\
PT on the day of the invasive procedure & 1370 & 15,341 \\
Total & 7056 & 81,262 \\
(2) Post-invasive procedure costs & \\
PT at $\geq 1$ day after the invasive procedure & 117,446 & 96,603 \\
Other than PT & & 30,732 \\
Total & 5162 & 659,022 \\
$(1)+(2)$ & 608,612 & 689,754 \\
\hline
\end{tabular}

All costs are average costs per patient presented in Japanese yen ( $\$ 1.00$ corresponds to approximately $¥ 109.67$ as of August 2021)

LUSU lusutrombopag, $P T$ platelet transfusion

${ }^{a}$ Prophylactic treatment costs: costs for prophylactic treatments to increase platelet counts, including those for lusutrombopag prescribed within 30 days prior to the invasive procedure and PT prescribed at 1 day before and on the day of the invasive procedure

${ }^{b}$ Post-invasive procedure costs: total medical costs between the day of the invasive procedure and 30 days after the invasive procedure, excluding those for $\mathrm{PT}$ on the day of the invasive procedure

compared. However, compared with PT, treatment with lusutrombopag has previously shown preferable effectiveness in maintaining $[5,7,10]$ or increasing platelet counts $[11]$, and the fact that lusutrombopag is convenient to use is worth noting. In addition, the safety and effectiveness of lusutrombopag in real world settings were indicated in a recent post-marketing surveillance study [16]. Taken together, lusutrombopag is possibly a desirable prophylactic treatment against the reduction of platelet counts in patients with CLD undergoing planned invasive procedures; therefore, it could be an alternative therapy to PT.

There are several limitations in this study. Only a small number of patients had platelet counts recorded in this database, and we were not able to determine thrombocytopenic patients based on the platelet counts. Thus, it was assumed that patients who received treatment with lusutrombopag or PT for the planned invasive procedures were thrombocytopenic patients. In addition, we were not able to match the groups based on platelet counts before the invasive procedure, which may have been associated with the occurrence of bleeding events. Therefore, we used other information related to the characteristics of patients, including age, type of invasive procedure, length of hospitalization before the invasive procedure, presence of SLD, and medical costs before the invasive procedure, to match the groups. More accurate results could have been obtained if the platelet counts before the invasive procedure were used for matching.

This study included patients who underwent invasive procedures at 5-30 days from the prescription of a course of treatment with lusutrombopag in the lusutrombopag group. According to the Japanese package insert of lusutrombopag, it should be started at 8-13 days prior to an invasive procedure and 


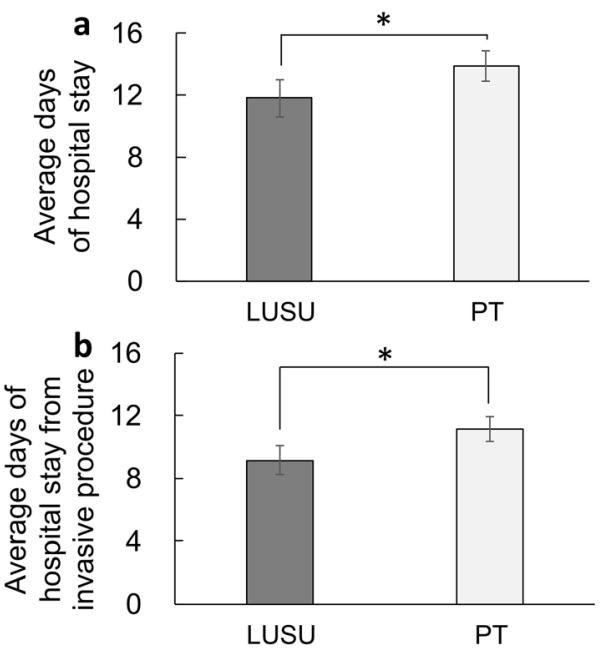

Fig. 3 Length of hospital stay as the average number of total hospitalization days for the invasive procedure (a) and the average number of days from the day of the invasive procedure until the day of discharge (b). Error bars represent $95 \%$ confidence intervals. LUSU lusutrombopag, PT platelet transfusion; ${ }^{*} p<0.05$

prescribed for 7 days [8]. Nonetheless, we selected 5 days as the minimum duration between the initiation of treatment with lusutrombopag and planned invasive procedures based on data from a clinical trial in which the platelet counts exceeded $50 \times 10^{9} / 1$ on the 5th day of lusutrombopag administration [5]. We selected 30 days as the maximum by considering the duration between the most recent outpatient visit before an invasive procedure and the invasive procedure in actual clinical practice in Japan. In the database, the prescription date, but not the initial date on which the drug was taken, could be identified. If the lusutrombopag group was limited to patients who took lusutrombopag in strict compliance with the guidance on the package insert, a more accurate evaluation may have been possible.

The characteristics of the database may have also caused a limitation. It consisted of data from only DPC hospitals. Thus, treatments, diagnoses, and medical costs in medical institutes other than DPC hospitals could not be included in the analysis. This may not have a considerable effect on this study, as most medical practices could have been included because of the relatively short observation period, which was 30 days before the invasive procedure until the day of hospital discharge or a maximum of 30 days after the invasive procedure and included the period of hospitalization. Moreover, as clinical practices vary among countries, some results of this study using the Japanese database, including the length of hospital stay and medical costs, could be different from those in other countries and areas.

\section{CONCLUSION}

Claims data analysis showed that among patients with CLD, the number of bleeding events associated with planned invasive procedures was lower in the lusutrombopag group than in the PT group. In addition, patients receiving lusutrombopag had reduced medical costs and length of hospital stay. Therefore, treatment with lusutrombopag can potentially be an alternative prophylactic treatment to increase platelet counts in thrombocytopenic patients with CLD who are scheduled to undergo planned invasive procedures.

\section{ACKNOWLEDGEMENTS}

Funding. Shionogi \& Co., Ltd., provided sponsorship for this study, including payment for the Journal's Rapid Service and Open Access fees.

Medical Writing and/or Editorial Assistance. Editage (http://www.editage.com) provided editorial assistance in preparing this article, and support for this assistance was funded by Shionogi \& Co., Ltd.

Authorship. All authors named in this article meet the International Committee of Medical Journal Editors (ICMJE) criteria for authorship, take responsibility for the integrity of the work as a whole, and provide their approval for the manuscript version to be published. 
Author Contributions. Manami Yoshida, Ryosuke Tateishi, Shinzo Hiroi, Yoshie Hongo, Masakazu Fujiwara, Yoshitake Kitanishi, and Ataru Igarashi contributed to study conception. All authors contributed to study design and data interpretation. Kosuke Iwasaki contributed to data analysis. Manami Yoshida and Tomomi Takeshima drafted the manuscript, and all authors revised it critically for important intellectual content. All authors approved the manuscript version to be submitted and have agreed both to be personally accountable for the authors' own contributions and to ensure that questions related to the accuracy or integrity of any part of the work, even ones in which the author was not personally involved, are appropriately investigated and resolved, with the resolution documented in the literature.

Prior Presentation. Parts of this work were presented at the 30th Asian Pacific for The Study of Liver (APASL) 2021 (virtual meeting) held on February 4-6, 2021.

Disclosures. Manami Yoshida, Shinzo Hiroi, Yoshie Hongo, and Yoshitake Kitanishi are employees of Shionogi \& Co., Ltd. Ryosuke Tateishi declares no competing interests. Masakazu Fujiwara was an employee of Shionogi \& Co., Ltd. atthe time the study was conducted. Masakazu Fujiwara is currentlyaffiliated with AstraZeneca KK, Osaka, Japan. Kosuke Iwasaki and Tomomi Takeshima are employees of Milliman Inc., which has received consultancy fees from Shionogi \& Co., Ltd. Ataru Igarashi has received consulting fees and lecture fees from GSK Japan Inc., MSD Inc., Pfizer Japan Inc., Sanofi-Pasteur Inc., Novartis Pharma K.K., AbbVie GK, Astellas Pharma Inc., Chugai Pharmaceutical Co. Ltd., Asahi Kasei Pharma Inc., Novo Nordisk Pharma Inc., Eisai Inc., Beckton Dickinson and Company, Maruho Co. Ltd., Ono Pharmaceutical Inc., Sato Pharmaceutical Inc., Sumitomo Dainippon Pharma Inc., and Eli Lilly Japan K.K., and research grants from Taiho Pharmaceutical Co. Ltd., GSL Behring Japan Inc., Gilead Science K.K., Fuji Film K.K., Terumo Corpration., Takeda Pharmaceutical Inc., Boston Scientific Japan Inc., DeSC Healthcare Inc.,
Otsuka Pharmaceutical K.K., Varian Medical Systems Inc., and Intuitive Surgical GK.

Compliance with Ethics Guidelines. This study was approved by Ethics Committee at the University of Tokyo. All procedures followed were in accordance with the ethical standards of Ethical Guidelines for Medical and Health Research Involving Human Subjects by Ministry of Education, Culture, Sports, Science and Technology, and Ministry of Health, Labour and Welfare and with the Helsinki Declaration of 1975, as revised in 2008. Since the database consisted of anonymized data collected for secondary use, informed consent was not required.

Data Availability. The datasets generated during and/or analyzed during the current study are available from the corresponding author on reasonable request and with permission from Medical Data Vision Co., Ltd.

Open Access. This article is licensed under a Creative Commons Attribution-NonCommercial 4.0 International License, which permits any non-commercial use, sharing, adaptation, distribution and reproduction in any medium or format, as long as you give appropriate credit to the original author(s) and the source, provide a link to the Creative Commons licence, and indicate if changes were made. The images or other third party material in this article are included in the article's Creative Commons licence, unless indicated otherwise in a credit line to the material. If material is not included in the article's Creative Commons licence and your intended use is not permitted by statutory regulation or exceeds the permitted use, you will need to obtain permission directly from the copyright holder. To view a copy of this licence, visit http://creativecommons.org/licenses/by$\mathrm{nc} / 4.0 /$.

\section{REFERENCES}

1. Afdhal N, McHutchison J, Brown R, et al. Thrombocytopenia associated with chronic liver disease. J Hepatol. 2008;48(6):1000-7. 
2. Peck-Radosavljevic M. Thrombocytopenia in chronic liver disease. Liver Int. 2017;37(6):778-93.

3. Hayashi H, Beppu T, Shirabe K, Maehara Y, Baba H. Management of thrombocytopenia due to liver cirrhosis: a review. World J Gastroenterol. 2014;20(10):2595-605.

4. Takami A, Matsushita T, Ogata M, et al. Guideline for the use of platelet transfusion concentrates based on scientific evidence: update 2019. Jpn J Transfus Cell Ther. 2019;65(3):544-61.

5. Hidaka H, Kurosaki M, Tanaka H, et al. Lusutrombopag reduces need for platelet transfusion in patients with thrombocytopenia undergoing invasive procedures. Clin Gastroenterol Hepatol. 2019;17(6):1192-200.

6. Brown RS, Bentley R, Cai B, et al. The clinical and economic burden of patients with chronic liver disease and thrombocytopaenia receiving platelet transfusions during planned invasive procedures. GastroHep. 2019;1(5):236-48.

7. Tateishi R, Seike M, Kudo M, et al. A randomized controlled trial of lusutrombopag in Japanese patients with chronic liver disease undergoing radiofrequency ablation. J Gastroenterol. 2019;54(2):171-81.

8. Mulpleta Tablets 3mg [Package Insert]. Shionogi \& Co., Ltd. December, 2015. https://www.info.pmda. go.jp/go/pdf/340018_3399010F1022_1_03. Accessed 27 Jan 2021. (Japanese).

9. Yoshiji H, Nagoshi S, Akahane T, et al. Evidencebased clinical practice guidelines for liver cirrhosis 2020. Hepatol Res. 2021;51(7):725-49.
10. Peck-Radosavljevic M, Simon K, Iacobellis A, et al. Lusutrombopag for the treatment of thrombocytopenia in patients with chronic liver disease undergoing invasive procedures (L-PLUS 2). Hepatology. 2019;70(4):1336-48.

11. Furuichi $Y$, Takeuchi H, Yoshimasu Y, Kasai Y, Abe M, Itoi $\mathrm{T}$. Thrombopoietin receptor agonist is more effective than platelet transfusion for chronic liver disease with thrombocytopenia, shown by propensity score matching. Hepatol Res. 2020;50(9):1062-70.

12. About MDV Database: Medical Data Vision Co. Ltd. https://en.mdv.co.jp/about-mdv-database/. Accessed 4 Aug 2020.

13. Anatomical Classification: The European Pharmaceutical Market Research Association. https://www. ephmra.org/classification/anatomicalclassification/. Accessed 4 Aug 2020.

14. Statistical classification of diseases and cause of death 2013: Ministry of Health, Labour and Welfare. http://www.mhlw.go.jp/toukei/sippei/. Accessed 4 Aug 2020.

15. Instruction manual of project to promote studies on liver cancer and severe cirrhosis treatment (for medical institution): document collection 2021: Ministry of Health, Labor and Welfare. https:// www.mhlw.go.jp/content/10900000/000763068. pdf. Accessed 30 July 2021. (Japanese).

16. Sasaki R, Shiino C, Imawari $M$, et al. Safety and effectiveness of lusutrombopag in Japanese chronic liver disease patients with thrombocytopenia undergoing invasive procedures: interim results of a postmarketing surveillance. Hepatol Res. 2019;49(10):1169-81. 